This item was submitted to Loughborough's Research Repository by the author.

Items in Figshare are protected by copyright, with all rights reserved, unless otherwise indicated.

\title{
Tomographic sensing of displacement fields
}

PLEASE CITE THE PUBLISHED VERSION

http://dx.doi.org/10.1364/SENSORS.2013.SW3D.2

PUBLISHER

(C) The Optical Society (OSA)

VERSION

AM (Accepted Manuscript)

LICENCE

CC BY-NC-ND 4.0

REPOSITORY RECORD

Ruiz, Pablo D.. 2019. "Tomographic Sensing of Displacement Fields". figshare.

https://hdl.handle.net/2134/14041. 
This item was submitted to Loughborough's Institutional Repository (https://dspace.lboro.ac.uk/) by the author and is made available under the following Creative Commons Licence conditions.

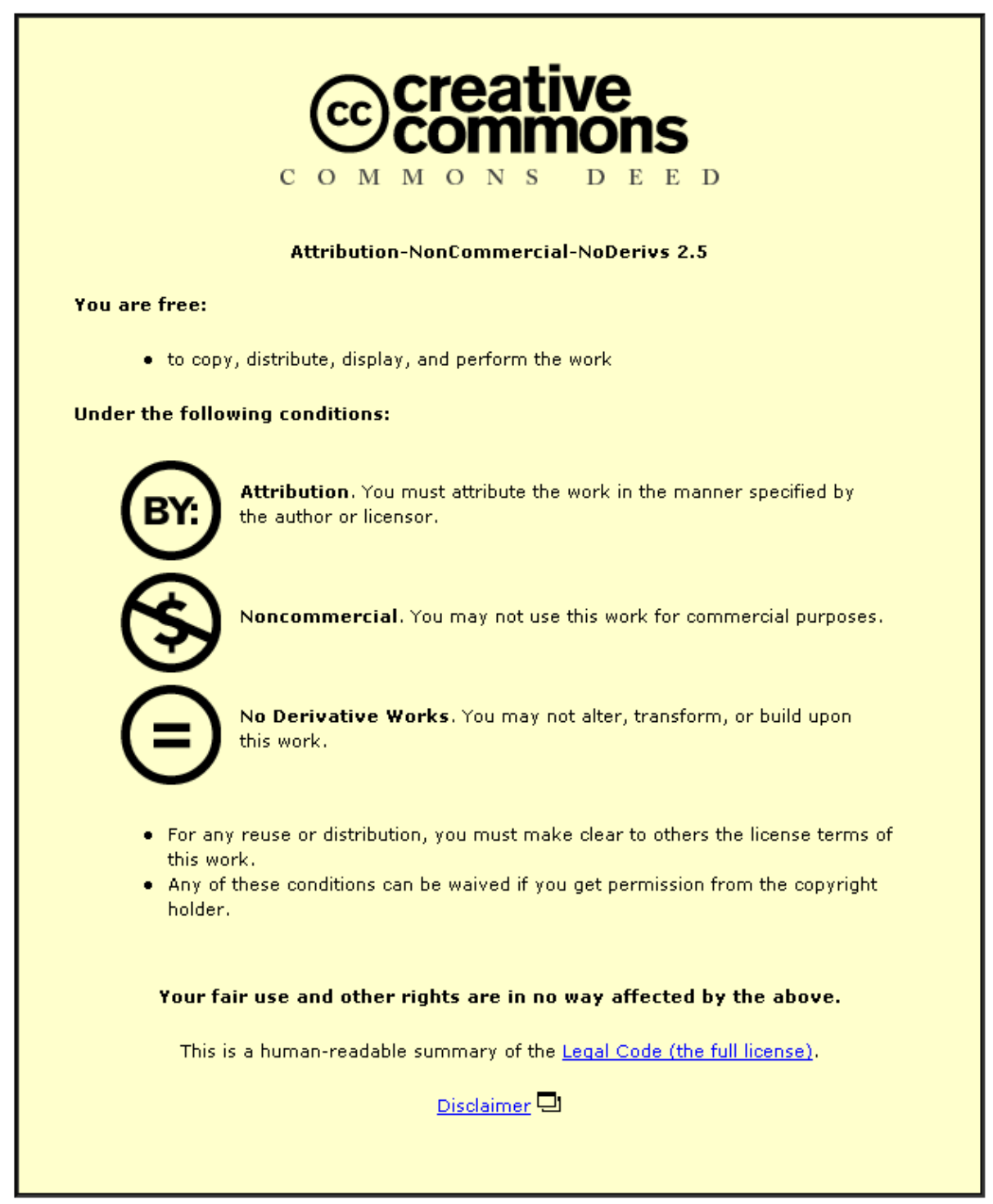

For the full text of this licence, please go to: http://creativecommons.org/licenses/by-nc-nd/2.5/ 


\title{
Tomographic sensing of displacement fields
}

\author{
Pablo D. Ruiz \\ Loughborough University, Ashby Road, Loughborough, United Kingdom, LE11 $3 T U$ \\ p.d.ruiz@lboro.ac.uk
}

\begin{abstract}
A wavelength scanning interferometry system is used to measure all the orthogonal components of the displacement field inside semitransparent scattering materials. A near infrared tunable laser illuminates a sample from multiple directions. The image of the sample is recombined with a reference beam on a photodetector array. As the laser frequency is linearly tuned during a scan, a sequence of speckle interferograms is recorded. In order to reconstruct the sample structure, Fourier transformation is performed on a pixel by pixel basis along the temporal axis of the 3-D data cube obtained. Multiple displacement sensitivities are achieved by introducing different optical delays between the reference and the illumination beams, which separate the reconstruction signals in the frequency domain. Phase changes due to mechanical loading of the sample can finally be measured and combined to obtain all orthogonal components of the displacement field in a convenient coordinate system. Controlled rigid body rotations of an epoxy phantom have been used to validate the methodology.
\end{abstract}

OC IS codes: (120.0120) Instrumentation, measurement, and metrology; (120.3180) Interferometry; (120.4290) Nondestructive testing; (120.5050) Phase measurement; (120.6160) Speckle interferometry

\section{Introduction}

Mechanical characterization of materials often involves the measurement of the physical dimensions of a sample, the forces acting on it and the displacements/strains produced by the load. Parameters such as the elastic modulus, Poisson's ratio and stiffness matrix components can then be determined. The resistive strain gauge is perhaps the most ubiquitous strain sensor in a mechanical engineering laboratory and provides the average strain in a specified direction under the area of the gauge, of the order of $1-10 \mathrm{~mm}^{2}$. However, multiple measurements require multiple sensors, which have to be glued to the sample as they rely on physical contact to transfer surface strain to the gauge. Fibre Bragg sensors constitute the optical counterpart of the resistive strain gauge and can be arranged along a single optical fibre as a one-dimensional (1-D) sensor array addressed through the same fibre, which is usually glued to a surface on embedded in the material under study. A broad range of alternative techniques have evolved to provide strain measurements with higher dimensionality and in a non-contact way. For instance, speckle interferometry and digital image correlation can both provide 2-D displacement and strain fields over opaque surfaces [1]. Depth resolved techniques go a step further and provide information of the material under the surface. Whereas neutron diffraction can provide strain fields through the thickness of crys talline materials such as metals, magnetic resonance imaging can be adapted to measure mechanical deformation of spin rich materials such as tissues containing water and fat [2]. X-ray computed tomography relies on attenuation to reconstruct the material structure and is combined with digital volume correlation (DVC) to evaluate 3-D displacement and strain fields [3]. In the optical domain, optical coherence tomography (OCT) can also provide depth-resolved displacement measurements either by using phase information [4,5], polarization sensitive detection and material photoelasticity [6], or detecting changes in the microstructure using digital volume correlation, known as OCT elastography [7]. In this paper, a novel approach is presented in which all the orthogonal components of the displacement field are measured simultaneously using wavelength scanning interferometry (WSI)[8]. Each voxel in the digital reconstruction of the sample behaves effectively as a multicomponent displacement sensor.

\section{Wavelength Scanning Interferometry}

WSI, also known as frequency scanning interferometry, or parallel Fourier-domain/swept-source OCT, fundamentally consists of an interferometer that uses a tunable laser as the light source (although other types of tunable sources exist). The illumination and observation directions define the sensitivity vectors that link the measured phase changes with the sought after displacements. A sequence of interferograms is recorded as the laser frequency is linearly scanned across the laser tuning range. Depth information along the observation direction is encoded for each pixel in the temporal frequency of the interference signal. The sample microstructure can thus be reconstructed by Fourier transformation of that signal along the time axis. High modulation frequencies correspond to scatterers that lay deep under the sample's surface, whereas low frequencies correspond to shallow ones. The phase and magnitude of the optical field can be extracted from the complex data provided by the Fourier transform. 
Displacements along the sensitivity directions are then obtained simply from the phase difference evaluated between a 'reference' and a 'deformed' state of the sample. Inversion of the sensitivity matrix then leads to the calculation of the displacement field in a Cartesian coordinate system. In a WSI system, the depth resolution is the axial (along the observation direction) distance between two scatterers that can be resolved using a Rayleigh criterion. It is inversely proportional to the spectralbandwidth of the light source. The depth range is the maximum depth that can be reconstructed and is inversely proportional to the laser frequency step between successive frames during image acquisition. Even though out-of-plane and in-plane OCT systems have been reported before $[4,5]$, full sensitivity systems that provide the out-of-plane and both in-plane displacement components remained elusive. One solution to that problem consists of using multiple out-of-plane WSI systems with different illumination/observation directions. Another alternative, and the one described in this paper, consists in multiple illumination directions and a single observation direction. In order to separate the microstructure reconstructions in the frequency domain, a different carrier frequency is used for each illumination direction by simply setting different optical delays betwe en them and the reference beam - see Ref. [8] for details. Figure 1 illustrates the optical setup:Light from a tunable laser (TSL510 Type A, Santec Ltd., tunable from $1260 \mathrm{~nm}$ to $1360 \mathrm{~nm}$ in $\sim 0.011 \mathrm{~nm}$ steps ) is split with a $2 \times 4$ fibre splitter in three illumination and one reference beams. The illumination beams are non-coplanar and collimated in order to achieve uniform sensitivity to all orthogonaldirections across the field of view. The sample is imaged by a $4 f$ system onto an InGaAs near infrared detector array (SU640SDV-1.7RT/RS 170, Goodrich Corporation, 640×512 pixels, 14 bits) where it interferes with the reference beam. In order to test the performance of the systemto measure 3-D displacement fields, a semitransparent thick epoxy plate was mounted on two independent rotation stages to introduce an in-plane rotation and an out-of-plane tilt. These displacement fields have constant gradients and are effective for validation purposes.

Figure 2 shows the displacement fields obtained in different cross sections of the sample. The second, third and fourth rows correspond to horizontal, vertical and out-of-plane $(u, v, w)$ displacements, respectively. An optical path axis $\Lambda=2 n z$ is used instead, where $z$ is the depth axis and $n$ the refractive index of the material. The columns show different cross sections through the measurement volume. Due to unwrapping errors in the phase components, an error-free data cube of size $0.6 \times 0.4 \times 0.8 \mathrm{~mm}^{3}$ was obtained, containing $16 \times 35 \times 71$ voxels. The lack of uniformity in the displacement fields is due to speckle, which average size was $\sim 0.05 \times 0.05 \times 0.07 \mathrm{~mm}^{3}$. The root mean squared deviation between the expected and measured displacements in the $x, y$ and $z$ directions were $0.14 \mu \mathrm{m}, 0.20$ $\mu \mathrm{m}$ and $32.5 \mathrm{~nm}$, respectively.

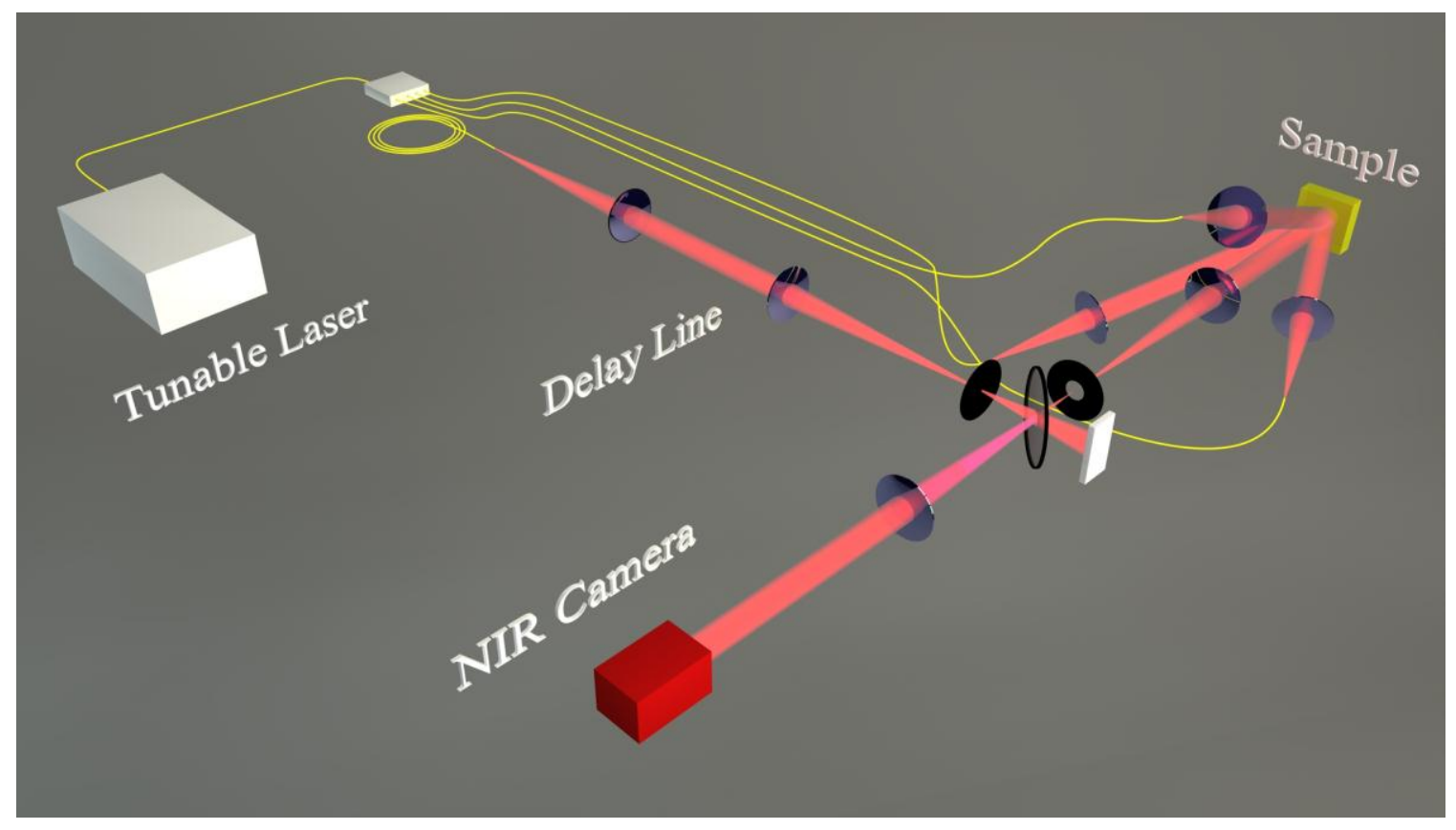

Fig. 1. WSI Mach-Zehnder interferometer with multipleillumination directions that arefrequency multiplexed by offsetting their optical path delays. 


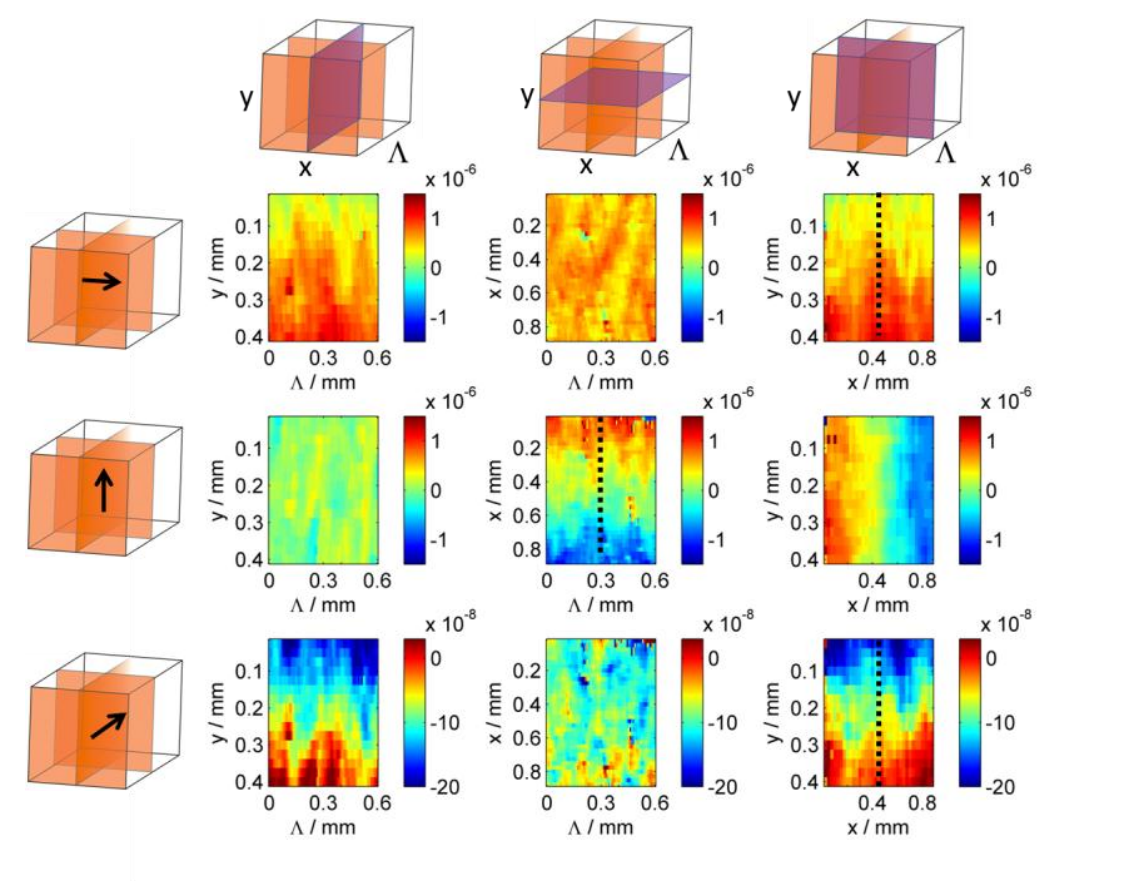

Fig. 2. Cross sections of the measured 3-D displacement field corresponding to a sample under in-plane rotation and out-of-plane tilt.

\section{Conclusion}

A new approach to provide 3-D displacement fields with high sensitivity inside the volume of semitransparent scattering materials has been presented. It does not rely on material birefringence (as in polarization sensitive OCT) to provide strain information, as they can be computed using numerical differentiation of the 3-Ddisplacement field.

Some issues that require further investigation in order to obtain large data volumes with low noise and small position and displacement uncertainties include robust 3-D unwrapping, refraction distortion of the structure reconstruction due to a curved sample surface, dispersion compensation, effect of birefringence, and speckle reduction. Exiting opportunities lie ahead in the field of characterization of materials such as composites and tissues.

\section{References}

[1] H. J. Tiziani, and G. Pedrini, "From speckle pattern photography to digital holographic interferometry," Appl. Opt., 52, 30-44 (2013).

[2] Y. Feng, T. M. Abney, R. J. Okamoto et al., "Relative brain displacement and deformation during constrained mild frontal head impact," Journal of the Royal Society Interface, 7, 1677-1688 (2010).

[3] B. K. Bay, T. S. Smith, D. P. Fyhrie et al., "Digital volume correlation: three-dimensional strain mapping using X-ray tomography," ExperimentalMechanics, 39, 217-226(1999).

[4] M. H. De la Torre-Ibarra, P. D. Ruiz, and J. M. Huntley, "Simultaneous measurement of in-plane and out-of-plane displacement fields in scattering media using phase-contrast spectral optical coherence tomography," Optics Letters, 34, 806-808 (2009).

[5] M. H. De la Torre-Ibarra, P. D. Ruiz, and J. M. Huntley, "Double-shot depth-resolved displacement field measurement using phase-contrast spectral optical coherence tomography," Optics Express, 14, 9643-9656(2006).

[6] D. Stifter, "Beyond biomedicine: a review of alternative applications and developments for optical coherence tomography," Applied Physics B-Lasers and Optics, 88,337-357 (2007).

[7] J. M. Schmitt, “OCT elastography: imaging microscopic deformation and strain oftissue,” Optics Express, 3, 199-211 (1998).

[8] S. Chakraborty, and P. D. Ruiz, "Measurement of all orthogonal components of displacement in the volume of scattering materials using wavelength scanning interferometry,"JOSA A, 29, 1776-1785,(2012). 